Gynäkol Geburtshilfliche Rundsch 1993;33(Suppl 1):172-173

\title{
Management des Gestationsdiabetes
}

$\mathrm{R}$

Univ. Frauenklinik München-Großhadern (Vorst.: Prof. Dr. H. Hepp)

PD. Dr. R. Knitza, Frauenklinik im Klinikum Großhadern der LMU, Marchionistraße 15, D-8000 München 70

In der Zeit vom 1. 1.84 bis 1. 1.92 wurden an der Universitäts-Frauenklinik im Klinikum Großhadern, Mün-chen insgesamt 380 Schwangere mit Gestationsdiabetes behandelt. Von diesen 380 Frauen benötigten 22\% eine Insulinbehandlung. Die Sectiorate des Gesamtkollektivs betrug $31,5 \%$.

Wenngleich ein allgemeines Screening auf eine dia-betische Stoffwechsellage an unserer Klinik derzeit nicht durchgeführt wird und nicht gut durchführbar ist, da nur ein geringer Bruchteil der Patientinnen von Beginn der Schwangerschaft an bei uns in Betreuung ist, wird die In-dikation zum Screening sehr großzügig bei anamnesti-schen und/oder klinischen Hinweise auf eine Kohlen-hydratverwertungsstörung gestellt (Tab. 1).

Tabelle 1. Screening bei folgenden Hinweisen

Diabetes in der Familie

Kind über $4.000 \mathrm{~g}$ Geburtsgewicht

Sonographisch großes Kind über der 90. Perzentile

Kind mit Mißbildungen

Wiederholte Frühgeburten/Aborte

Ungeklärter perinataler Kindesverlust in vorangegangener Schwangerschaft

Hydramnion

Kopf-Thorax-Diskrepanz

Glukosuri

EPH-Gestose

Rezidivierende Harnwegsinfektionen

Adipositas

Zeitpunkt des Screenings ist bei anamnestischen Hinweisen etwa die 24 .

Schwangerschaftswoche. Bei klinischen Hinweisen wird grundsätzlich unabhängig vom Zeitpunkt der Schwangerschaft eine Diagnostik einge-leitet. Aus Gründen der Praktikabilität führen wir zu-nächst entsprechend den Empfehlungen der American Diabetes Association und der Diabetic Pregnancy Study

Group der Europäischen Diabetesgesellschaft [1] ein Vorscreening mit Bestimmung des 1 Stunden-Wertes un-ter Belastung mit $50 \mathrm{~g}$ Glukose bei der nicht nüchternen Patientin durch. Als Grenzwert sehen wir eine Blutzuk-kerkonzentration (Vollblut von $130 \mathrm{mg} / \mathrm{dl}(7,2 \mathrm{mmol} / \mathrm{l})$ ) an. Wird dieser Wert überschritten, so führen wir den sogenannten ,großen oGTT” mit $100 \mathrm{~g}$ Glukose bei der nüchternen Patientin nach kohlenhydratreicher Vorer-nährung entsprechend den Empfehlungen der Deutschen Diabetesgesellschaft [2] durch. Als obere Grenzwerte der Blutglukose im Kapillarblut sehen wir einen Nüchtern-blutzuckerwert von über $90 \mathrm{mg} / \mathrm{dl}$, einen 
60-Minuten-Wert über $165 \mathrm{mg} / \mathrm{dl}$, einen 120 Minuten-Wert über $145 \mathrm{mg} / \mathrm{dl}$, und einen 180 Minuten-Wert über $125 \mathrm{mg} / \mathrm{dl}$ an. Bei zwei oder mehr Werten, die diese Grenzwerte überschreiten, erfolgt eine weitere Abklärung durch ein Blutzuckertagesprofil. Dieses Blutzuckertagesprofil wird ambulant nach entsprechender Beratung der Patientin über einige Tage geführt, wobei die Blutzuckerbestim-mungen zu folgenden Zeitpunkten erfolgen sollten: 1 . Bestimmung Nüchternwert morgens

Bestimmung

Bestimmung

Bestimmung

Bestimmung

Bestimmung

Parallel zur profile erfolgt

60 bis 90 Minuten nach dem Frühstück vor dem Mittagessen 60 bis 90 Minuten nach dem

Mittagessen

vor dem Abendessen 60 bis 90 Minuten nach dem Abendessen.

Durchführung dieser Blutzuckertages-eine individuelle Diätberatung der Schwangeren durch geschultes Personal. Ziel ist es, die Nahrungsaufnahme auf drei Haupt- und drei Zwischenmahlzeiten aufzuteilen, wobei der Energiebedarf mit etwa $35 \mathrm{kcal} / \mathrm{kg}$ Körpergewicht angesetzt wird. 40\% der zugeführten Energie sollten in Form von Kohlenhydra-ten, 25\% als Eiweiß und $35 \%$ als Fett erfolgen. Falls un-

ter einer derartigen Diättherapie zwei oder mehr Blutzuckerwerte im Tagesverlauf den Wert von $130 \mathrm{mg}$ über-schreiten, sehen wir eine Indikation zur Insulintherapie.

Die Betreuung der schwangeren Gestationsdia-betikerin erfolgt wenn irgend möglich ambulant, in zwei-wöchentlichen Intervallen und Absprache mit dem nie-dergelassenen Facharzt. Der Patientin wird ein Blutzuk-kermeßgerät zur Verfügung gestellt und die Patientin er-lernt, die Blutzuckerbestimmungen eigenständig durch-zuführen. Eine stationäre Aufnahme erfolgt stets zu Be-ginn einer notwendigen Insulineinstellung, wobei die Ho-spitalisierungsdauer meist nicht mehr als drei Tage be-trägt. Lediglich bei etwaigen Komplikationen oder Pro-blemen mit der Blutzuckerselbstbestimmung ist eine Hospitalisierung unumgänglich.

Während der Schwangerschaft werden in regel-mäßigen Intervallen folgende Laborparameter bestimmt: HBA lc,

Eiweiß und Glukose im 24-Stunden-Urin, eine Mikroproteinanalyse im Urin sowie die

Kreatininclearance.

Außerdem erfolgt initial eine Augenhintergrund-untersuchung.

Ultraschalluntersuchungen werden anfangs vier-wóchentlich und im dritten Trimenon in 14tägigem Ab-stand durchgeführt.

CTG-Schreibungen erfolgen ab der 26. SSW im Rah-men der Vorstellung; ab 32. SSW ein- bis zweimal wö-chentlich.

$\mathrm{Ab}$ der 36. SSW wird einmal in der Woche ein Bela-stungstest in Form eines

Oxytocinbelastungstestes oder aber auch als Nipple-Stiumaltions-Testes durchgeführt sowie eine fetale Blutflußmessung.

Als Entbindungzeitpunkt wird der errechnete Termin angestrebt, wobei hier individuell vorgegangen wird.

Bei nicht insulinpflichtigen Gestationsdiabetikerinnen erfolgt kein spezielles Monitoring während der Geburt. 
Für insulinpflichtige Gestationsdiabetikerinnen und elektiver Geburt wählen wir folgendes Vorgehen.

Die nüchterne Patientin erhält morgens kein Insulin, die angestrebten Blutzuckerwerte sollten etwa $100 \mathrm{mg} \%$ betragen. Während der Geburt erfolgt eine Blutzuk-kerkontrolle in 1-2stündigen Intervallen, wobei durch die Zufuhr von 5\%iger Glukose mit einer Geschwindigkeit von $160 \mathrm{ml} / \mathrm{Stunde}$, entsprechend $8 \mathrm{~g} / \mathrm{l}$, sowie 2 Einheiten Altinsulin/Stunde separat i.v. dieser Blutzuckerwert re-guliert wird. Gelegentlich, insbesondere bei stärkeren

Blutzuckerschwankungen, infundieren wir zusätzlich eine 5\%ige Xylitlösung mit einer Infusionsgeschwindigkeit von $60 \mathrm{ml} /$ Stunde, entsprechend $3 \mathrm{~g} / \mathrm{Stunde}$, was einen günstigen antiketogenen Effekt zeigt.

Die Neugeborenen von diabetischen Müttern erhalten eine Frühfütterung mit Dextroneonat sowie zusätzlich zu jeder Mahlzeit $10 \mathrm{ml}$ Milch und $2 \mathrm{ml}$ Calciumglukonat $10 \%$ ig. Vor jeder Mahlzeit wird eine Blutzuckerbestim-mung durchgeführt, wobei als Zielgröße mindestens 45 $\mathrm{mg} \%$ erreicht werden sollten. Bleibt der Blutzucker trotz Dextroneonat unter $40 \mathrm{mg} \%$, so erfolgt eine Glu-koseinfusion mit 1O\%iger Lösung in einer Infusionsgeschwindigkeit von 5 $\mathrm{ml} / \mathrm{kg} /$ Stunde, entsprechend $8 \mathrm{mg} / \mathrm{kg} /$ Minute [3].

Als Analgesieverfahren bei vaginalen Entbindungen von Gestationsdiabetikerinnen empfehlen wir die groß-zügige Durchführung einer Periduralanästhesie mit Ka-theter. Folge dieser kontrollierten Analgesie ist eine Re-duktion streßbedingter metabolischer Veränderungen, so zum Beispiel ein wesentlich geringerer Anstieg von Glukose, Nichtesterfettsäuren, Ketonkörpern und Lac-tat [4].

Mit dem hier dargelegten Vorgehen in der Betreuung von Gestationsdiabetikerinnen haben wir durchwegs positive Erfahrungen machen können. Insbesondere die ambulant durchzuführende, regelmäßige Blutzuckerselbst-kontrolle und die Bereitstellung eines Blutzuckermeß-gerätes mit Speicherfunktion hat die mittlere stationäre Behandlungsdauer der insulinpflichtigen

Diabetikerinnen erheblich reduziert.

Derzeit werten wir die von den Patientinnen ge-speicherten Daten mit einem entsprechenden Software-programm nach den verschiedensten Funktion aus. Wir haben den Eindruck, daß die Visualisierung der gemes-senen Daten, die sich dann wesentlich besser mit den Patientinnen besprechen läßt, zu einem weiteren Motiva-tionsschub bezüglich einer optimalen Blutzuckereinstellung führt. Wir würden es daher sehr begrüßen, wenn in Deutschland die Kosten für die Anschaffung der Blut-zuckermeßgeräte mit Speicherfunktion in das Lei-stungsspektrum der Krankenkassen aufgenommen würde.

\section{Literatur}

Summary and Recommendations of the 2nd International Workshop-Conference on Ge-stational Diabetes Mellitus: Diabetes (34), Suppl. 2, 1985, 123-126.

Ellmann O, Gries SA, Hepp KD, Otto H, Pottroff S, Sauer H, Schöffling K, Weber B: Die ärztliche Führung der graviden Dia-betikerin. Stellungnahme der Deutschen Diabetesgesellschaft. Dtsch. Ärzteblatt 79 (1982) 37-38.

Versmold H: Richlinien Neonatologie. 5. Auf-lage 1988

Klinikum Großhadern,Ludwig-Maxi-milians-Universität München. Knitza R: Auswirkung geburtshilflicher Analgesieverfahren auf Geburtsverlauf

Geburts-erlebnis

hormonell- 
